ARTICLE

DOI: $10.1038 / \mathrm{s} 41467-017-00952-3$

\title{
Glycaemic control boosts glucosylated nanocarrier crossing the BBB into the brain
}

\author{
Y. Anraku1, H. Kuwahara ${ }^{2,3}$, Y. Fukusato ${ }^{1}$, A. Mizoguchi ${ }^{4}$, T. Ishii ${ }^{1}$, K. Nitta ${ }^{2,3}$, Y. Matsumoto ${ }^{4,5}$, K. Toh $^{6}$, \\ K. Miyata ${ }^{4,7}$, S. Uchida1,4, K. Nishina2,3, K. Osada', K. Itaka ${ }^{4}$, N. Nishiyama ${ }^{8}$, H. Mizusawa 2,3, T. Yamasoba ${ }^{5}$, \\ T. Yokota 2,3 \& K. Kataoka6,9
}

Recently, nanocarriers that transport bioactive substances to a target site in the body have attracted considerable attention and undergone rapid progression in terms of the state of the art. However, few nanocarriers can enter the brain via a systemic route through the bloodbrain barrier (BBB) to efficiently reach neurons. Here we prepare a self-assembled supramolecular nanocarrier with a surface featuring properly configured glucose. The BBB crossing and brain accumulation of this nanocarrier are boosted by the rapid glycaemic increase after fasting and by the putative phenomenon of the highly expressed glucose transporter-1 (GLUT1) in brain capillary endothelial cells migrating from the luminal to the abluminal plasma membrane. The precisely controlled glucose density on the surface of the nanocarrier enables the regulation of its distribution within the brain, and thus is successfully optimized to increase the number of nanocarriers accumulating in neurons.

\footnotetext{
${ }^{1}$ Department of Bioengineering, Graduate School of Engineering, The University of Tokyo, 7-3-1 Hongo, Bunkyo-ku, Tokyo 113-8656, Japan. ${ }^{2}$ Department of Neurology and Neurological Science, Graduate School of Medical and Dental Sciences, Tokyo Medical and Dental University (TMDU), 1-5-45 Yushima, Bunkyo-ku, Tokyo 113-8519, Japan. ${ }^{3}$ Center for Brain Integration Research, Tokyo Medical and Dental University (TMDU), 1-5-45 Yushima, Bunkyo-ku, Tokyo 113-8519, Japan. ${ }^{4}$ Center for Disease Biology and Integrative Medicine, Graduate School of Medicine, The University of Tokyo, 7-3-1 Hongo, Bunkyo-ku, Tokyo 113-0033, Japan. ${ }^{5}$ Department of Otorhinolaryngology and Head and Neck Surgery, Graduate School of Medicine and Faculty of Medicine, The University of Tokyo, 7-3-1 Hongo, Bunkyo-ku, Tokyo 113-0033, Japan. ${ }^{6}$ Innovation Center of NanoMedicine, Kawasaki Institute of Industrial Promotion, 3-2514 Tonomachi, Kawasaki-ku, Kawasaki 210-0821, Japan. ${ }^{7}$ Department of Materials Engineering, Graduate School of Engineering, The University of Tokyo, 7 3-1 Hongo, Bunkyo-ku, Tokyo 113-8656, Japan. ${ }^{8}$ Laboratory for Chemistry and Life Science, Institute of Innovative Research, Tokyo Institute of Technology, R1-11, 4259 Nagatsuta, Midori-ku, Yokohama 226-8503, Japan. ${ }^{9}$ Policy Alternatives Research Institute, The University of Tokyo, 7-3-1 Hongo, Bunkyo-ku, Tokyo 113-0033, Japan. Y. Anraku, H. Kuwahara and Y. Fukusato contributed equally to this work. Correspondence and requests for materials should be addressed to T.Y. (email: tak-yokota.nuro@tmd.ac.jp) or to K.K. (email: kataoka@bmw.t.u-tokyo.ac.jp)
} 
R ecent advances in nanotechnology have allowed the development of nanocarriers based on the controlled selfassembly of precisely designed molecules as drug vehicles with various different shapes and sizes ${ }^{1-7}$. These nanocarriers are a promising system for delivering therapeutic or diagnostic agents to diseased sites in the body, particularly to solid tumours with highly permeable blood vessels ${ }^{8-10}$. In fact, clinical trials of nanocarriers for tumour-targeted delivery are currently in progress $^{11-14}$. Nevertheless, designing nanocarriers that transport biologically active substances through the barrier of poorly permeable blood vessels remains a challenging task. In particular, the brain is strongly protected by the blood-brain barrier (BBB), a system consisting primarily of brain capillary endothelial cells (BCECs) along with pericytes and astrocytes ${ }^{15,16}$. Only small hydrophobic molecules with a molecular weight lower than $\sim 450$ can penetrate the $\mathrm{BBB}^{15}$. Because the intractability of many central nervous system (CNS) disorders arises from the BBB inhibiting the delivery of various drugs into the brain, developing a method of using nanocarriers to efficiently deliver drugs through the BBB is of the highest priority. Many efforts towards transporting nanocarriers into the brain have been reported to date. A recent example is a BBB-crossing nanocarrier equipped with peptides capable of recognizing the transferrin receptor on BCECs; however, its accumulation rate in the brain did not exceed $1.0 \%$ dose/g-brain ${ }^{17}$. In another example, apolipoprotein E-bound nanoparticles crossed the BBB to reach neurons, but the delivery efficiency was not reported ${ }^{18}$. Among the various candidate ligands previously studied for promoting BBB traversal, glucose, the main energy source in the brain, is notable because glucose transporter-1 (GLUT1) is expressed at a remarkably high level compared to many other receptors and transporters in BCECs ${ }^{19}$. Although several nanocarriers targeting GLUT1 by incorporating glucose as the ligand have been designed ${ }^{20,21}$, none of them have been transported into the brain at a high level. Therefore, the GLUT1-based delivery strategy requires an innovative approach.

Here we report a biological strategy that exploits the rapid glycaemic increase after fasting to enable a remarkably enhanced delivery of a nanocarrier across the BBB via GLUT1.

\section{Results}

Preparation of glucose-integrated nanocarriers. GLUT1 recognizes glucose molecules principally through weak interactions such as hydrogen bonding and the hydrophobic effect $\left(K_{\mathrm{m}}=1.5 \mathrm{mM}\right)^{22}$. Therefore, the binding of a single glucose to GLUT1 may not be strong enough to retain nanocarriers in the harsh flow of the bloodstream. A promising strategy to achieve strong retention of nanocarriers on BCECs through GLUT1 binding is to induce a multivalent interaction between a nanocarrier and multiple GLUT1 molecules on BCECs (polyvalent effect $^{23,24}$ ) by introducing many glucose molecules onto the a
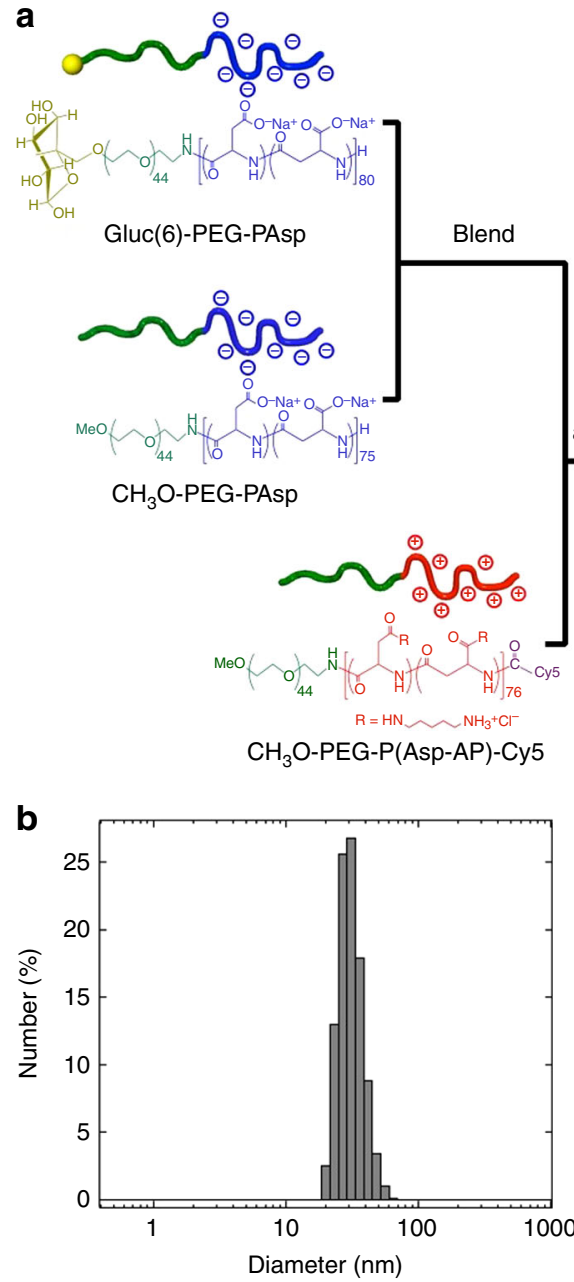

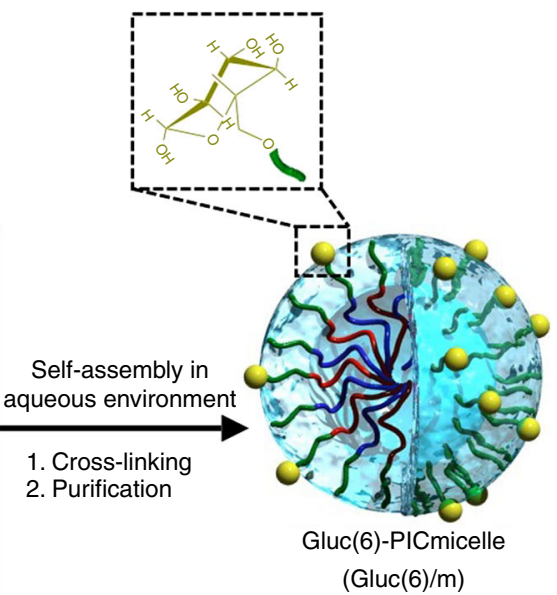

C

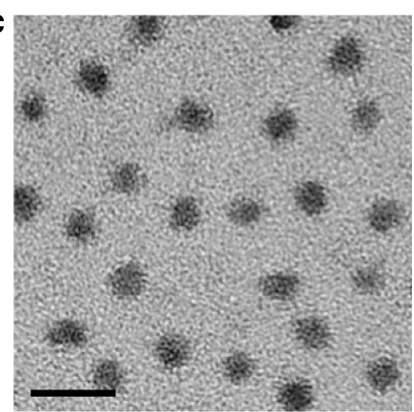

Fig. 1 Characterization of the Gluc(6)-conjugated PIC micelle (Gluc(6)/m). a Scheme of Gluc(6)/m preparation via the assembly of oppositely charged block copolymers. b Size distribution of the $25 \%$ Gluc(6)/m determined by DLS. c TEM image of the $25 \%$ Gluc(6)/m. The scale bar indicates $50 \mathrm{~nm}$ 
surface of the nanocarrier that is sufficiently larger in size than a single GLUT1 molecule $(\sim 3 \mathrm{~nm})^{25}$. For this purpose, we constructed a polymeric micellar nanocarrier with a size of $\sim 30 \mathrm{~nm}$ via the multimolecular association of oppositely charged pairs of polyethylene glycol (PEG)-based block ionomers. The surface of the polymeric micelles was decorated with multiple
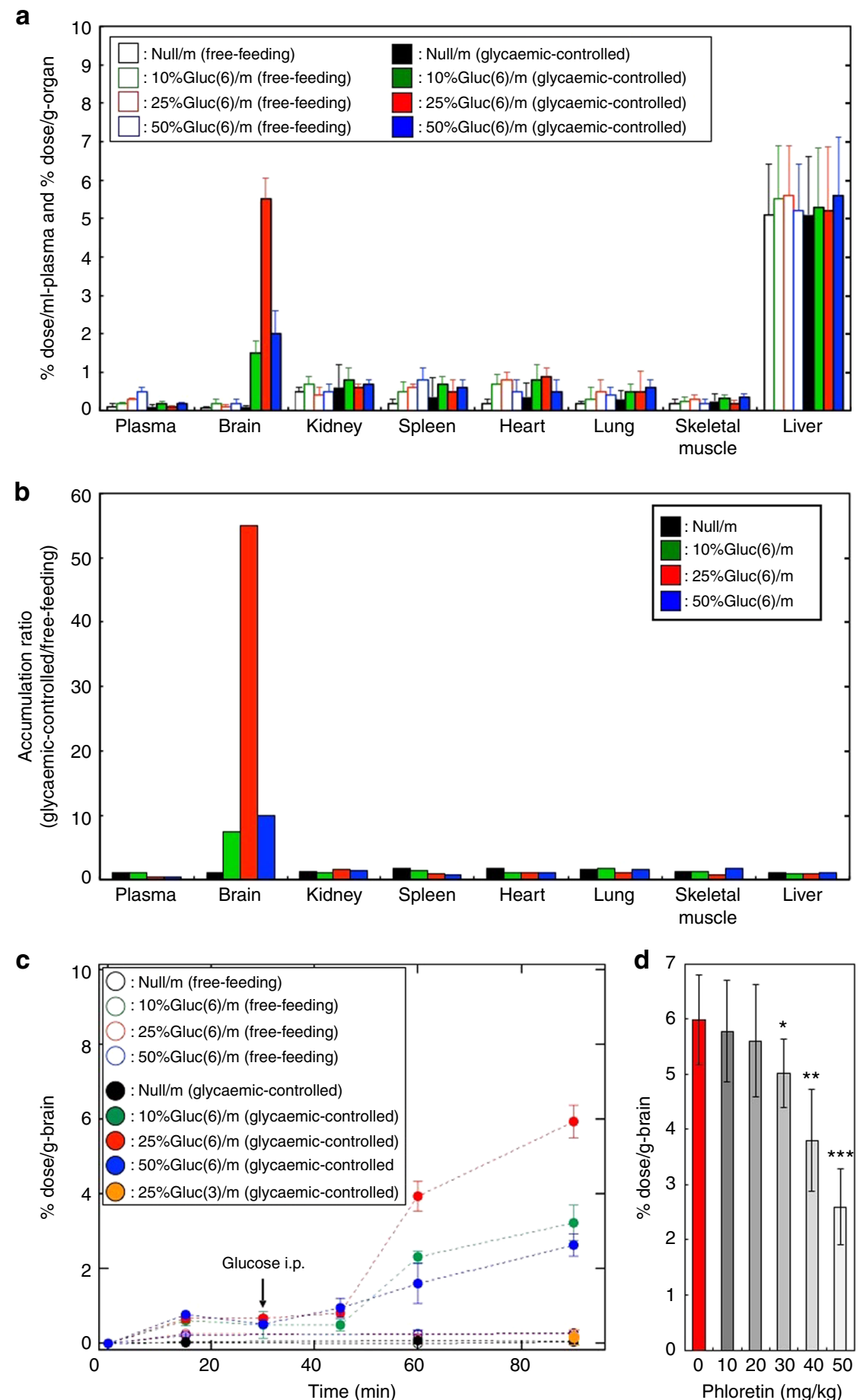

Fig. 2 Pharmacokinetic profiles of the polymeric micelles. a Biodistribution of micelles ( Null/m and Gluc(6)/m) in mice under different feeding conditions at $48 \mathrm{~h}$ after the injection. Open and closed bars show free-feeding and glycaemic-controlled groups, respectively. Black, green, red and blue coloured bars indicate Null/m, 10\%Gluc(6)/m, 25\%Gluc(6)/m and 50\%Gluc(6)/m, respectively. b Accumulation ratio in mice (glycaemic-controlled/free-feeding) of each micelle at $48 \mathrm{~h}$ calculated from the values shown in a. c Time course of micelle accumulation (Null/m, Gluc(6)/m and Gluc(3)/m) in the mouse brain under different feeding conditions. The arrow in c indicates when $20 \mathrm{wt} \%$ glucose was intraperitoneally injected. Open and closed circles denote the freefeeding and glycaemic-controlled groups, respectively. Black, green, red and blue coloured circles indicate Null/m, 10\%Gluc(6)/m, 25\%Gluc(6)/m and $50 \%$ Gluc(6)/m, respectively. An orange coloured circle shows $25 \% \mathrm{Gluc}(3) / \mathrm{m}$ (glycaemic-controlled). d In vivo inhibition study of the brain accumulation of the $25 \% \mathrm{Gluc}(6) / \mathrm{m}$ by phloretin. Red and grey bars show experiments conducted without and with phloretin, respectively. Experiments were performed using BALB/c mice (female, 6-week-old, $n=5$ ). The data are expressed as the mean \pm SEM for $\mathbf{a}, \mathbf{c}$, $\mathbf{d}$ and as the mean for $\mathbf{b}$. ${ }^{\star} P<0.05$ 
glucose molecules with a controlled density (Fig. 1a). Glucose was introduced onto the micelles via an ether linkage at the C6 position to preserve the binding ability to GLUT1 because the $\mathrm{OH}$ groups at positions $\mathrm{C} 1, \mathrm{C} 3$ and $\mathrm{C} 4$ of glucose are believed to be essential for its interaction with GLUT1 ${ }^{26}$. Specifically, a PEG$\operatorname{poly}(\alpha, \beta$-aspartic acid) block copolymer (PEG-PAsp) with glucose conjugated to the $\alpha$-end of the PEG segment via a bond with the C6-O moiety (Gluc(6)-PEG-PAsp) $\left(M_{\mathrm{n}}\right.$ of PEG $=2000$, DP of $\mathrm{P}(\mathrm{Asp})=80)$ was blended in an aqueous solution at an arbitrary proportion with $\mathrm{CH}_{3} \mathrm{O}-\mathrm{PEG}-\mathrm{PAsp}\left(M_{\mathrm{n}}\right.$ of $\mathrm{PEG}=2000$, DP of $\mathrm{P}$ $($ Asp $)=75)$ (blending ratio of Gluc(6)-PEG-PAsp to $\mathrm{CH}_{3} \mathrm{O}-\mathrm{PEG}$ $\mathrm{PAsp}=1: 0,1: 1,1: 4$ and 0:1). This solution was then mixed with an aqueous solution of oppositely charged $\mathrm{CH}_{3} \mathrm{O}-\mathrm{PEG}-$ poly $([5-$ aminopentyl]- $\alpha, \beta$-aspartamide) block copolymer $\left(M_{\mathrm{n}}\right.$ of $\mathrm{PEG}=$ 2000, DP of $\mathrm{P}($ Asp-AP $)=76$ ) labelled with a fluorescence dye $(\mathrm{Cy} 5)$ at the $\omega$-end $\left(\mathrm{CH}_{3} \mathrm{O}-\mathrm{PEG}-\mathrm{P}(\mathrm{Asp}-\mathrm{AP})-\mathrm{Cy} 5\right)$ at a charge ratio of $1: 1$ and stabilized by crosslinking with 1-ethyl-3(3-dimethylaminopropyl) carbodiimide. In this manner, a series of polyion complex (PIC) micelles consisting of a crosslinked PIC core covered by a hydrophilic, non-charged PEG shell with a constant size and systemically altered surface glucose densities (Gluc(6)-conjugated PIC micelles (X\%Gluc(6)/m, X=10, 25 and 50)) were prepared (Fig. 1a). For the control micelles that may not bind to GLUT1, PIC micelles without the surface glucose $(\mathrm{Null} / \mathrm{m})$ and those with glucose conjugated via an ether linkage at the C3 position $(\mathrm{Gluc}(3) / \mathrm{m})$ were prepared. Dynamic light scattering (DLS) measurement and transmission electron microscopy (TEM) observation revealed that the PIC micelles are monodisperse (polydispersity index $<0.1$ ) with a diameter of 30 $\mathrm{nm}$ regardless of the glucose density $(0,10,25$ or $50 \%)$ or the conjugating position of glucose (Gluc(6) or Gluc(3)) (Fig. 1b, c, Supplementary Fig. 12 and Supplementary Table 1).

Next, we assessed the binding property of the PIC micelles to GLUT1 in vitro. For this purpose, we generated a GLUT1overexpressing stable cell line by transfecting mouse GLUT1expressing plasmid (Supplementary Fig. 13A, B), added the micelles with or without the surface glucose to the medium of these cells on a culture plate and quantified their uptake into the cells by measuring the fluorescent intensities of the detached cell suspension. The uptake into GLUT1-overexpressing cells, not into mock-transfected cells, was increased for the $\operatorname{Gluc}(6) / \mathrm{m}$ (Supplementary Fig. 13C), and this increase of uptake was completely inhibited by the standard GLUT1 inhibitors cytochalasin $B$ and phloretin, which have different mechanisms of action (Supplementary Fig. 13D). The cellular uptake of the Gluc(3)/m was comparable to that of the Null/m, excluding the possibility of a nonspecific sugar-mucosal interaction (Supplementary Fig. 13C). These data indicate that the glucose molecules attached via the C6 position onto the micelle surface indeed can bind to mouse GLUT1 expressed on the cell membrane.

Glycaemic control boosts nanocarrier accumulation in the brain. We next examined the pharmacokinetic profiles of our polymeric micelles upon intravenous administration into normal free-feeding mice. All micelles irrespective of the presence of surface glucose showed prolonged retention in blood: more than $80 \%$ of the initial dose remained in the blood circulation $90 \mathrm{~min}$ after an intravenous injection (Supplementary Fig. 14). At $48 \mathrm{~h}$ after injection, the accumulation of the micelles in most organs, including the brain, was very limited to below $1 \%$ dose/g-organ, even for the Gluc(6)/m (Fig. 2a, open bars). The accumulation in the liver was up to $5-6 \%$ dose/g-liver, and the accumulation was not increased by decorating with glucose (Fig. 2a, open bars), suggesting that the liver uptake of the micelles was mostly by a nonspecific mechanism. These results indicate that the strategy of only decorating the nanocarriers with glucose does not have a substantial effect on the delivery of polymeric micelles into the brain.

We then considered introducing glycaemic control with the animal model. Lowering the blood glucose concentration was reported to facilitate GLUT1 localization on the luminal plasma membrane in BCECs ${ }^{27}$; therefore, GLUT1 would be internalized into BCECs based on an elevation of blood glucose concentration. Based on this assumption, we investigated the effect of glycaemic control on the accumulation of our micelles in the brain by intravenously injecting the micelles into mice after a 1-day fast and intraperitoneally administering a $20 \mathrm{wt} \%$ glucose solution 30 min after the micelle injection to elevate the blood glucose concentration. The accumulation of the micelles in most organs regardless of the presence of the surface glucose was unchanged by introducing this glycaemic control. However, a remarkable and specific increase in the micelle accumulation in the brain was observed in only the Gluc(6)/m-administered mice (Fig. 2a, b, closed bars). The surface glucose density of the Gluc(6)/m had substantial effect on accumulation, with the $25 \% \mathrm{Gluc}(6) / \mathrm{m}$ exhibiting the highest accumulation rate of up to $6 \% \mathrm{dose} / \mathrm{g}$ brain (Fig. 2a, red closed bar), which was 56 times higher than the rate observed in free-feeding mice (Fig. 2b, red closed bar). An accumulation rate of $6 \%$ dose/g-brain is appealing because it is significantly higher than those reported to date for other carrier systems using glucose or peptide ligands ${ }^{17,20,21}$ and is almost equal to the enhanced permeability and retention effect (EPR)driven accumulation rate of long-circulating stealth nanocarriers into tumours with highly leaky vasculatures ${ }^{1,8,9}$.

An evaluation of the time course revealed that when the $20 \mathrm{wt}$ $\%$ glucose solution was administered to fasting mice subsequent to the micelle injection, a remarkable increase in the micelle accumulation into the brain starting $\sim 15 \mathrm{~min}$ after the glucose administration was observed only in the Gluc(6)/m-administered mice (Fig. 2c). Importantly, Gluc(3)/m failed to achieve a similarly remarkable accumulation in the brain (the accumulation rate was comparable to that of Null/m (0.2\%)) after the same glycaemic control (Fig. 2c). This notable difference in the brain accumulation rate between $\operatorname{Gluc}(6) / \mathrm{m}$ and $\mathrm{Gluc}(3) / \mathrm{m}$ strongly suggests the involvement of GLUT1 because the C1, C3 and C4$\mathrm{OH}$ groups in glucose are essential for the recognition by GLUT1 ${ }^{26}$. As C3-OH in Gluc(3)/m was substituted with an ether linkage to PEG, Gluc(3)/m is assumed to have low affinity towards GLUT1. Moreover, the involvement of GLUT1 was suggested by the considerable decrease in the accumulation of $25 \%$ Gluc(6)/m in the brain (Fig. 2d) upon intravenous administration of phloretin (a GLUT1 inhibitor).

Although there was a previous study on the brain delivery of glucose-decorated PEGylated liposomes, no glycaemic manipulation of fasting animals was conducted and the accumulation rate was limited to only $0.4 \%$ dose/g-brain ${ }^{20}$. This result is consistent with our results obtained for the mice that were not intraperitoneally administered glucose ( $0.3 \%$ dose/g-brain), suggesting that the manipulation of blood glucose concentration is essential for achieving significant and selective accumulation of glucoseintegrated nanocarriers into the brain.

Direct observation of nanocarriers crossing the BBB. After establishing the outstanding accumulation of $25 \% \mathrm{Gluc}(6) / \mathrm{m}$ in the brain, we attempted to directly observe the micelles crossing the $\mathrm{BBB}$ using intravital real-time confocal laser-scanning microscopy (IVRT-CLSM). IVRT-CLSM allows spatiotemporal and quantitative analysis of behaviours such as the extravasation and tissue crossing of nanocarriers in a living animal ${ }^{28} .25 \%$ Gluc (6)/ $\mathrm{m}$ was injected intravenously into fasting mice, followed by an 
a
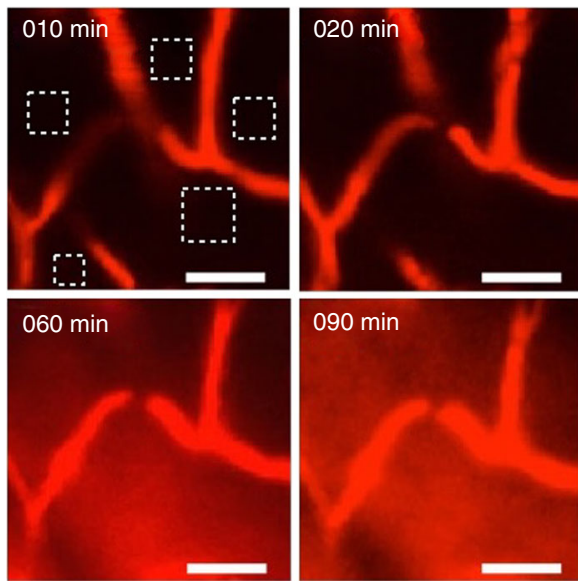

c
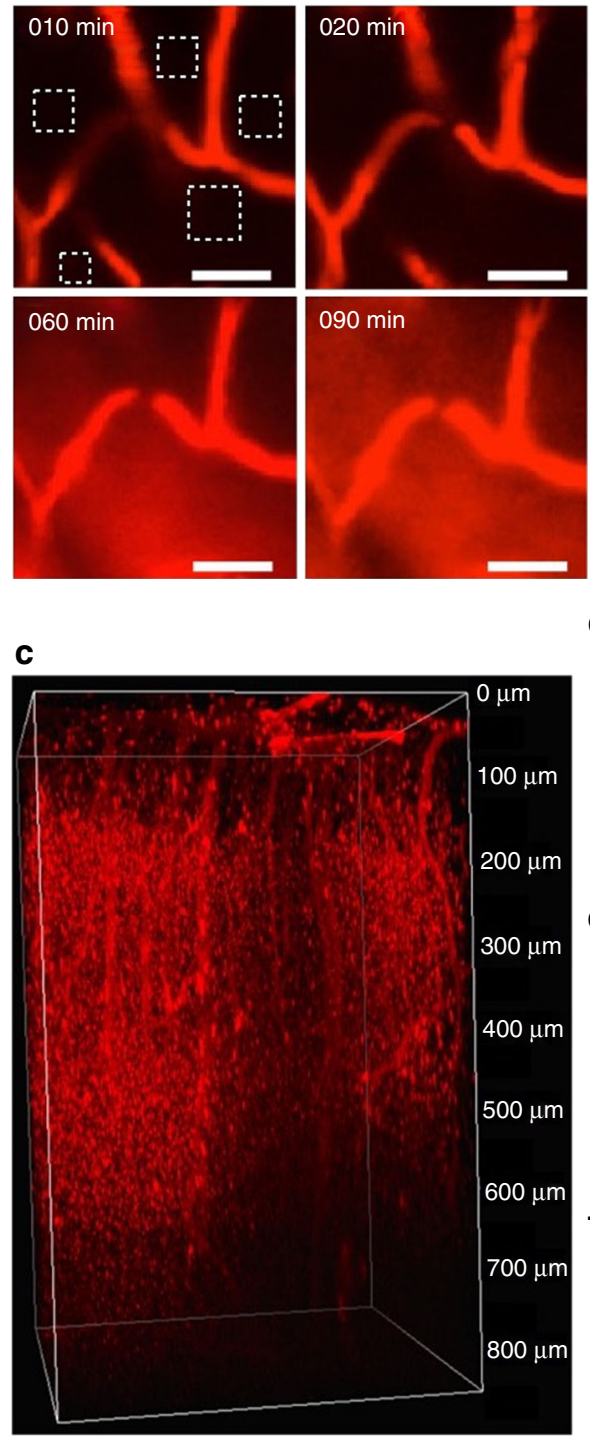

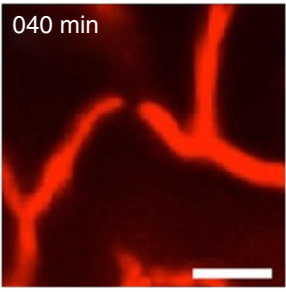

$120 \mathrm{~min}$
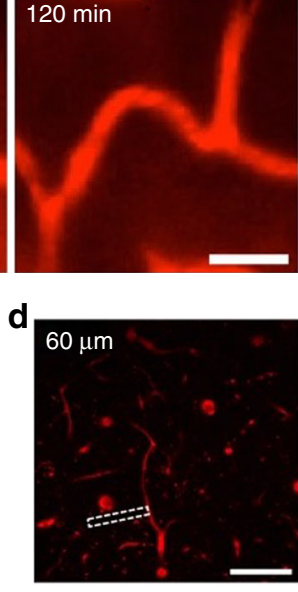

e $300 \mu \mathrm{m}$
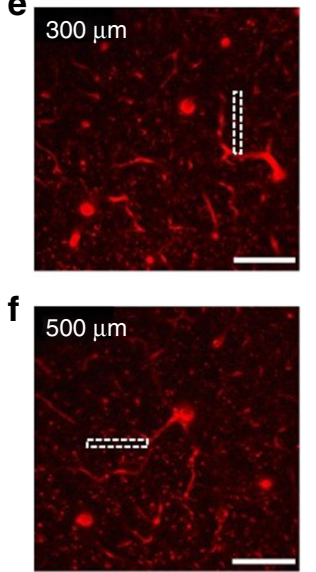
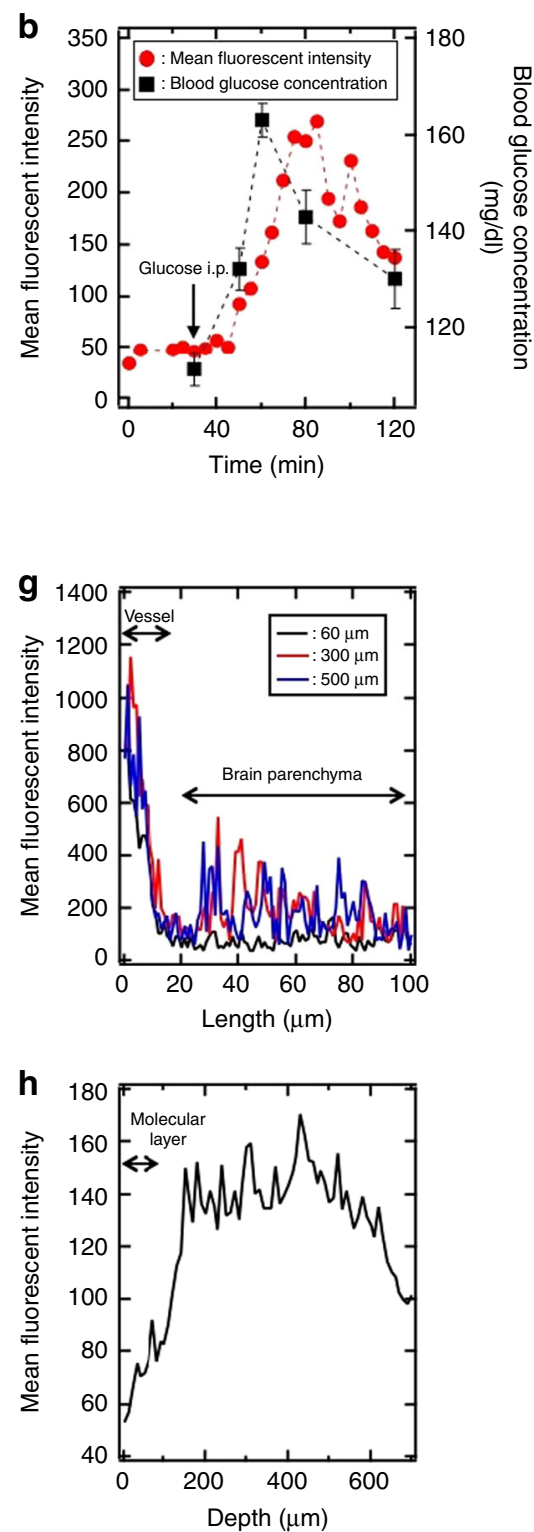

Fig. 3 Real-time observation of the $25 \%$ Gluc(6)/m crossing the BBB. a Sequential images of mouse cerebrum observed using IVRT-CLSM. The 25\%Gluc (6)/m (red) were intravenously injected to a mouse after a 24-h fast, followed by an intraperitoneal injection of 20 wt $\%$ glucose 30 min later. The scale bars indicate $50 \mu \mathrm{m}$. b Time course of blood glucose concentration (black squares) and the mean fluorescent intensities of the $25 \% \mathrm{Gluc}(6) / \mathrm{m}$ (red circles) in the region of interest (ROI) of the brain parenchyma indicated as white rectangles in $\mathbf{a}$. The arrow indicates the time of intraperitoneal injection of the 20 wt\% glucose. Blood glucose concentrations were expressed as the mean \pm SEM. c Images of Gluc(6)/m (red) in the mouse cerebrum observed using intravital multiphoton microscopy $48 \mathrm{~h}$ after administration. Cross-sections at depths of $60 \mu \mathrm{m}(\mathbf{d}), 300 \mu \mathrm{m}(\mathbf{e})$ and $500 \mu \mathrm{m}(\mathbf{f})$ are also shown. The scale bars indicate $100 \mu \mathrm{m}$. $\mathbf{g}$ Distribution profiles of Gluc(6)/m from blood vessels to the brain parenchyma in the selected region (indicated by a white rectangle in $\mathbf{d}, \mathbf{e}, \mathbf{f}$ ) at depths of $60 \mu \mathrm{m}$ (black line), $300 \mu \mathrm{m}$ (red line) and $500 \mu \mathrm{m}$ (blue line). $\mathbf{h}$ Depth profiles of the mean fluorescent intensities from 0 to $700 \mu \mathrm{m}$ in the brain parenchymal region

administration of the $20 \mathrm{wt} \%$ glucose solution $30 \mathrm{~min}$ later. In this experiment, we evaluated the real-time extravasation of the micelles through the BBB based on the intensity and distribution of fluorescent signals. Figure 3a shows IVRT-CLSM micrographs of the cerebrum. Initially, at $10 \mathrm{~min}$ after the administration of the $25 \% \operatorname{Gluc}(6) / \mathrm{m}$ (before the administration of the glucose solution), fluorescent signals (red) were observed only inside of the vasculature. Next, at $60 \mathrm{~min}$ after the administration of the $25 \%$ Gluc $(6) / \mathrm{m}$ (30 min after the administration of the glucose solution), the fluorescent signals were observed extensively throughout the brain parenchyma (the areas apart from the bloodstream) and then further increased in intensity until $90 \mathrm{~min}$. To quantitatively evaluate the micelles crossing the BBB, five different regions in the brain parenchyma were selected (squares enclosed by white dotted lines in Fig. 3a), and the fluorescent intensities were measured until $120 \mathrm{~min}$ (Fig. 3b, red circles). The blood glucose concentration was monitored at 30, 50, 60, 80 and $120 \mathrm{~min}$ after administering the $25 \%$ Gluc(6)/m (Fig. 3b, black squares). The fluorescent intensities in the brain parenchyma started to increase at $\sim 10-20 \mathrm{~min}$ after the injection of glucose 


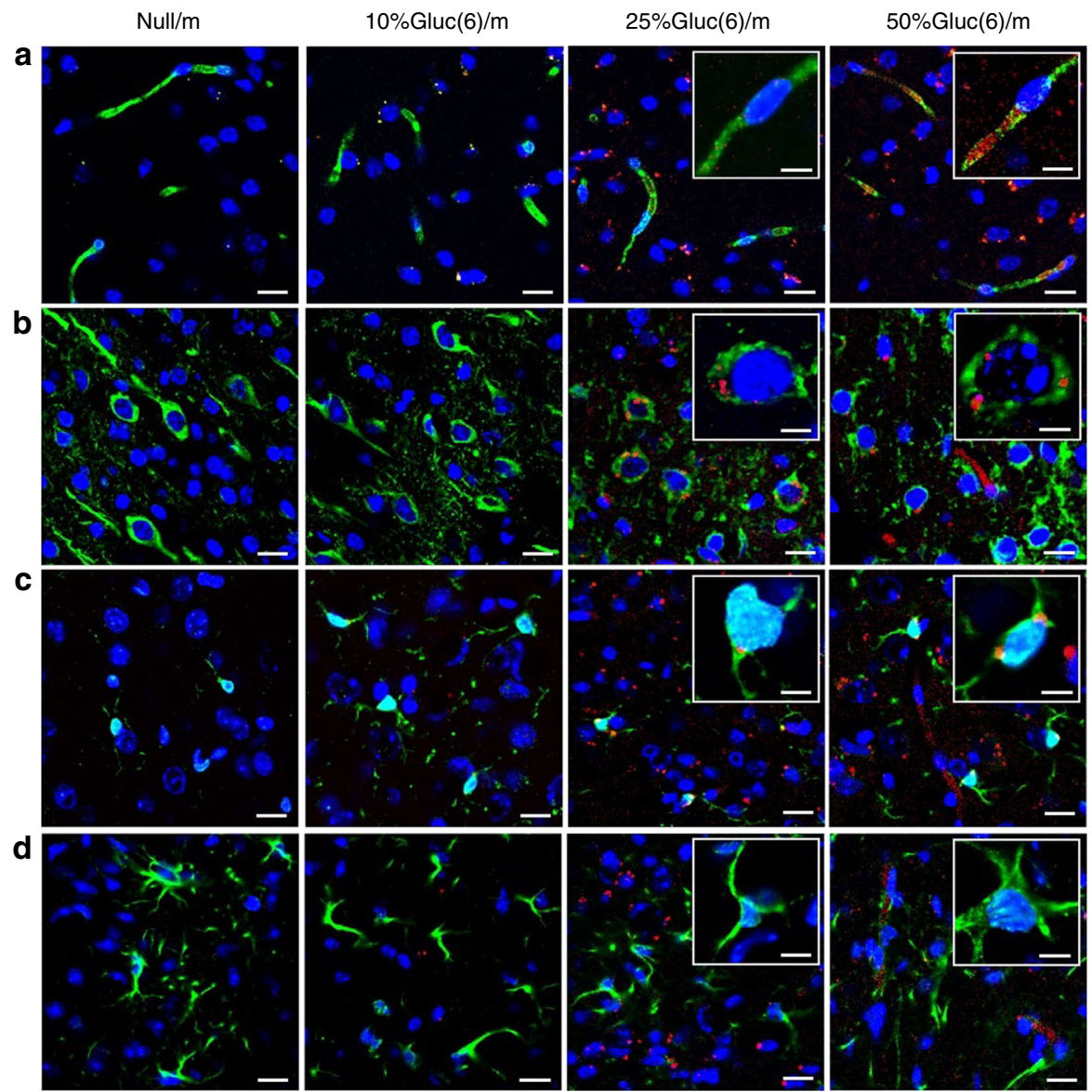

Fig. $4 \mathrm{Imm}$ unohistochemical analysis of the mouse brains after administration of polymeric micelles. Cerebral sections at $48 \mathrm{~h}$ after the administration of Null/m, 10\%Gluc(6)/m, 25\%Gluc(6)/m and 50\%Gluc(6)/m (red). BCECs (a), neurons (b), microglia (c) and astrocytes (d) (green) are stained with antiPECAM1, anti-Tuj1, anti-lba1 and anti-GFAP antibodies, respectively. Nuclei (blue) are stained with DAPI. The scale bar indicates $20 \mu \mathrm{m}(10 \mu \mathrm{m}$ in insets)

solution, i.e., as the blood glucose concentration increased. The decay of the fluorescent intensities after the peak also closely followed the decrease in the blood glucose concentration.

Notably, at $48 \mathrm{~h}$ after the micelle administration, intravital multiphoton microscopy clearly showed the fluorescent signals of the $25 \% \operatorname{Gluc}(6) / \mathrm{m}$ in the extravascular parenchyma of the cerebrum at a depth of up to $700 \mu \mathrm{m}$ from the cortical surface (Fig. 3c and Supplementary Movie 1). The cross-section images showed only slight $25 \% \operatorname{Gluc}(6) / \mathrm{m}$ distribution in the molecular layer where cellular components were scarce (Fig. 3d, g, h); by contrast, marked $25 \%$ Gluc(6)/m distribution was observed in the deeper layers where cellular components were abundant (Fig. 3e-h), suggesting that the micelles were taken up by parenchymal cells.

Distribution profiles of nanocarriers in the brain parenchyma. We then conducted immunohistochemical analysis of brain specimens to determine the destination of the micelles. For this purpose, we first intravenously administered Null/m, 10\%Gluc $(6) / \mathrm{m}, 25 \%$ Gluc $(6) / \mathrm{m}$ or $50 \%$ Gluc $(6) / \mathrm{m}$ to fasting mice and then intraperitoneally injected them with $20 \mathrm{wt} \%$ glucose $30 \mathrm{~min}$ later. We obtained the mice brains $48 \mathrm{~h}$ after the administration of the micelles and stained the frozen sections with antibodies against PECAM1, Tuj1, Iba1 and GFAP to detect BCECs, neurons, microglia and astrocytes, respectively. The fluorescent signals of the micelles in the brain sections were then observed using confocal laser-scanning microscopy (Fig. 4). The Null/m were not recognized in any region of the brain. Although the $50 \% \operatorname{Gluc}(6) / \mathrm{m}$ were clearly distributed throughout the BCECs, the $10 \%$ Gluc(6)/m and $25 \%$ Gluc(6)/m were barely noticeable in this region (Fig. $4 \mathrm{a}$ ), suggesting that a considerable fraction of the $50 \%$ Gluc $(6) / \mathrm{m}$ stayed in and/or around the BCECs. An appreciable fraction of the $25 \%$ Gluc $(6) / \mathrm{m}$ and $50 \% \mathrm{Gluc}(6) / \mathrm{m}$ were incorporated into the neurons and microglia (Fig. 4b, c). As shown in Fig. 4d, we did not identify the accumulation of any micelles in the astrocytes regardless of the glucose density. The relatively higher accumulation of $50 \% \operatorname{Gluc}(6) / \mathrm{m}$ in the BCECs can be explained as a manifestation of their lower dissociation constant $\left(K_{\mathrm{d}}\right)$ to GLUT1 due to the high surface glucose density, which leads to the stacking of the Gluc(6)/m in and/or around the BCECs. Importantly, further investigations are needed to clarify the precise delivery route of Gluc(6)/m after crossing the BCECs, especially from the perspective of the micellar distribution within the neurovascular unit, which covers a wider range of components in the $\mathrm{CNS}^{29}$.

Delivery mechanism of the nanocarriers across the BCECs. Our study has revealed that the following three factors are critical for efficiently transporting glucose-integrated nanocarriers into the brain: (1) introducing multiple properly-oriented glucose molecules onto the surface of a polymeric micelle, (2) administering the micelle to fasting mice and (3) subsequently increasing blood glucose concentration.

In BCECs, GLUT1 is localized on both the luminal and abluminal plasma membranes, although the relative distribution is not uniform across species ${ }^{30}$. Additionally, GLUT1 is internalized via clathrin-independent endocytosis, and is translocated between intracellular compartments and the plasma 
membrane as a physiological phenomenon of intracellular recycling ${ }^{31-33}$, which is conceptually similar to the behaviour of glucose transporter-4 (GLUT4) in adipose and muscular cells in response to insulin 34,35 . The luminal plasma membrane levels of GLUT1 protein increase by more than $50 \%$ under hypoglycaemic conditions when compared with the levels under normal and hyperglycaemic conditions because of a redistribution that favours luminal transporters and an increase in GLUT1 synthesis at the gene expression level ${ }^{27}$. This phenomenon suggests that GLUT1 migrates from the cellular interior onto the plasma membrane surface of BCECs depending on the demand for glucose in the brain. Although there is no published evidence for GLUT1 translocation between the luminal and abluminal membrane in BCECs, the above characteristics of intracellular GLUT1 migration led us to the following interpretation: after attaching to GLUT1 on the luminal plasma membrane of BCECs, our nanocarrier was partly transcytosed through the BCECs to an abluminal site during the intracellular recycling of GLUT1 in response to a glycaemic change.

We then conducted immunohistochemical studies on the brain sections to obtain insight into the mechanism of the Gluc(6)/m crossing the BCECs by visualizing their recycling endosomes. For this purpose, we intravenously injected fasting mice with $25 \%$ Gluc $(6) / \mathrm{m}$ or $50 \%$ Gluc $(6) / \mathrm{m}$, then intraperitoneally administered them with glucose solution after $30 \mathrm{~min}$ and finally obtained the brains after an additional $30 \mathrm{~min}$ because we could estimate from the findings shown in Figs. 2 and 3 that the Gluc(6)/m were in the process of crossing the BCECs at this time point. Eventually, both $25 \% \mathrm{Gluc}(6) / \mathrm{m}$ and $50 \% \mathrm{Gluc}(6) / \mathrm{m}$ were partially localized at recycling endosomes immunolabelled with anti-Rab11a antibody (Supplementary Fig. 15A, B). When the glycaemic increase was not evoked, weak signals of the Gluc(6)/m were found within the BCECs, but no signals were localized in the recycling endosomes (Supplementary Fig. 15C, D). These observations are consistent with the proposed scheme that within BCECs, Gluc(6)/m may migrate together with the recycling endosomes in response to a glycaemic increase after a fasting state. Because the migration of GLUT4 within the intracellular recycling process takes $\sim 12-35 \mathrm{~min}^{35}$, we assume that the delay of 10-30 min between the elevation of blood glucose concentration and the accumulation of the Gluc(6)/m in the brain is consistent with our hypothesis that the delivery mechanism involves the intracellular recycling of GLUT1.

The intracellular migration of GLUT1 in BCECs must be controlled by multiple factors, including an endocrinological response and signal transduction within the neural circuit in relation to the blood glucose concentration, to maintain energy homoeostasis in vivo. A detailed physiological study would be necessary to fully explain the glycaemic-dependent delivery mechanism of our glucosylated nanocarriers across the BBB.

\section{Discussion}

We developed polymeric micelles decorated with properly configured glucose molecules to recognize GLUT1 on BCECs and successfully demonstrated their preferential and efficient crossing of the BBB induced by a glycaemic increase after a fasting state. The appeal of this system is the on-off switch delivery strategy, in which the transport of the ligand (glucose)-conjugated nanocarrier into the brain may be facilitated by an external trigger (glucose) inducing the intracellular recycling of transporter molecules (GLUT1). Indeed, the involvement of GLUT1 in the delivery of the glucose-conjugated polymeric micelles into the brain was verified experimentally, and the transport of the micelles through the BBB into the brain parenchyma was directly observed in situ under IVRT-CLSM. Furthermore, managing the glucose density on the polymeric micelle enabled cellular-level control over the destination in the brain: the $25 \% \mathrm{Gluc}(6) / \mathrm{m}$ are mostly directed to the neurons and microglia, whereas the $50 \%$ Gluc(6)/m primarily remain in and/or around the BCECs. These results suggest that the nanocarrier system reported here has potential for delivering various drugs directly into the brain by crossing the BBB. Nevertheless, in diseased conditions, GLUT1 in BCECs might have an altered expression level ${ }^{36}$ and/or might not similarly translocate from the luminal to abluminal plasma membrane. Additionally, in diseases such as glioma, the BBB integrity can be disrupted ${ }^{37}$. Thus, the clinical relevance of the present results should be further examined by using animal models that properly reflect the BBB structure in each disease and by optimizing the structure of the glucosylated nanocarriers and the condition of glycaemic control.

\section{Methods}

Materials and animals. Information regarding materials and animals is described in the "Methods" section of Supplementary Information. All animal experiments were performed in accordance with the Guidelines for the Care and Use of Laboratory Animals, as stated by the University of Tokyo and Tokyo Medical and Dental University.

Polymer synthesis. Detailed procedures for the preparation and characterization of $\mathrm{CH}_{3} \mathrm{O}-\mathrm{PEG}$-PAsp, $\mathrm{CH}_{3} \mathrm{O}-\mathrm{PEG}-\mathrm{P}(\mathrm{Asp}-\mathrm{AP})-\mathrm{Cy} 5$, Gluc(6)-PEG-PAsp and Gluc (3)-PEG-PAsp are provided in the Supplementary Figs. 1-11.

Preparation and characterization of polymeric micelles. Detailed preparation schemes of the Gluc(6)/m, Gluc(3)/m and Null/m are given in the Supplementary Fig. 12 and Supplementary Table 1. The size distribution of the polymeric micelles was evaluated by conducting DLS measurements at $25^{\circ} \mathrm{C}$ in $10 \mathrm{mM}$ phosphate buffer at pH 7.4 using a Zetasizer Nano ZS90 (Malvern Instruments Ltd., Worcestershire, UK). TEM images of the polymeric micelles were obtained with a JEM1400 (JEOL Ltd., Tokyo, Japan) according to the following sample preparation procedure: mesh copper grids were coated with a thin film of collodion followed by carbon coating, and a $0.1 \mathrm{mg} / \mathrm{ml}$ sample solution was placed on these carboncoated grids. The sample on the grid was stained with $2 \mathrm{wt} \%$ uranyl acetate in a mixture of water and ethanol $(\mathrm{v} / \mathrm{v}=1 / 1)$ and then blotted to remove any extra stain.

Biodistribution study. To analyse the distribution of the Gluc(6)/m, Gluc(3)/m and Null/m in vivo, BALB/c mice $(n=4)$ were reared without food for $24 \mathrm{~h}$. Then, these mice were intravenously injected with $200 \mu \mathrm{l} \mathrm{of} 1 \mathrm{mg} / \mathrm{ml} \mathrm{Cy5-labelled} \mathrm{poly-}$ meric micelle $(\operatorname{Gluc}(6) / \mathrm{m}$, Gluc(3)/m or Null/m) in D-PBS( -$)$ and, after $30 \mathrm{~min}$, intraperitoneally injected with $200 \mu \mathrm{l}$ of $20 \mathrm{wt} \% \mathrm{D}-(+)$-glucose solution in D-PBS(-). The mice were killed at 15,30, 45, 60 and $90 \mathrm{~min}$ and $48 \mathrm{~h}$ after the glucose injection and the excess blood was washed out by perfusion with D-PBS(-). The brain, liver, kidneys, spleen, heart, lung and femoral muscle were excised, washed with D-PBS(-), weighed after removing excess fluid and homogenized with cell lysis buffer. Blood was collected from the inferior vena cava, heparinized and centrifuged to obtain plasma. The accumulated amount of the micelles was quantified by fluorescence measurement using an Infinite M1000 PRO spectrophotometer (Tecan Group Ltd., Männedorf, Switzerland).

Effect of phloretin on brain accumulation of nanocarrier. To examine how the brain accumulation of the $25 \%$ Gluc(6)/m was effected by the inhibition of GLUT1, $\mathrm{BALB} / \mathrm{c}$ mice $(n=4)$ were fasted for $24 \mathrm{~h}$ and then intravenously injected with the solution of the $25 \% \mathrm{Gluc}(6) / \mathrm{m}$. After $30 \mathrm{~min}$, they were intraperitoneally injected with $200 \mu \mathrm{l}$ of $20 \mathrm{wt} \%$ glucose solution, followed by an intravenous injection of the GLUT1 inhibitor phloretin $(10-50 \mathrm{mg} / \mathrm{kg})^{38}$ at $20 \mathrm{~min}$ after glucose administration. The brain was extracted in the same manner as biodistribution study, and the accumulation of the $25 \% \mathrm{Gluc}(6) / \mathrm{m}$ in the brain was quantified by fluorescence measurement using the Infinite M1000 PRO (Tecan Group Ltd.).

\section{Intravital real-time confocal laser-scanning microscopy. The mice were} anaesthetized with $2.5 \%$ isoflurane (Abbott, North Chicago, IL) using a Univentor 400 anaesthesia unit (Univentor; Zetjun, Malta). The skulls of mice were partially cut open around the ROIs without damaging blood vessels. The mice were directly placed on a thermoplate (Tokai Hit, Tokyo, Japan), and a cover slip (Muto Pure Chemicals, Tokyo, Japan) was attached with proper pressure to flatten the brain surface. Images of the micelle crossing the BBB were acquired with an A1R confocal laser-scanning microscopy system (Nikon Corp., Tokyo, Japan) attached to an upright ECLIPSE FN1 (Nikon Corp.). 
Intravital multiphoton microscopy. Depth profile images of the micelle brain distribution were collected on a multiphoton microscope system equipped with a pulse laser (InSight DeepSee, Spectra Physics, Santa Clara, CA) and an Apochromat $\times 25$ water immersion objective lens $(2.0 \mathrm{~mm}$ WD, $1.10 \mathrm{NA}$ ) (Nikon A1R MP, Nikon Corp.). Imaging was performed using an excitation wavelength tuned at $1100 \mathrm{~nm}$. The mice were anaesthetized with $1.5-2 \%$ isoflurane, and the head was stabilized with a head holder (SGM-4, Narishige Co., Ltd., Tokyo, Japan). The scalp and dura were removed to create a $3-\mathrm{mm}$ diameter cranial window over the right hemisphere ( $2 \mathrm{~mm}$ lateral from the midline, $2 \mathrm{~mm}$ posterior to the bregma). After removing the skull bone, a glass bottom dish (diameter

$40 \mathrm{~mm}$, thickness $0.17 \mathrm{~mm}$, WillCo-dish ${ }^{\otimes}$, WillCo Wells B.V., Amsterdam, the Netherlands) was attached to the exposed brain tissue.

Immunohistochemical analysis of brain sections. The mice were deprived of food for $24 \mathrm{~h}$ and then intravenously injected with the solution of the Cy5-labelled polymeric micelles (Null/m, 10\%Gluc(6)/m, 25\%Gluc(6)/m and 50\%Gluc(6)/m, respectively), followed by an intraperitoneal injection of $20 \mathrm{wt} \%$ glucose solution. After $48 \mathrm{~h}$, the mice were killed and were perfused with PBS and $4 \%$ paraformaldehyde. The brains were fixed overnight in $4 \%$ paraformaldehyde at $4{ }^{\circ} \mathrm{C}$ and sequentially soaked overnight in $20 \mathrm{wt} \%$ sucrose solution at $4{ }^{\circ} \mathrm{C}$. The fixed specimens were snap-frozen in liquid nitrogen and then were sliced into $14-\mu \mathrm{m}$-thick sections using a CM3050 S cryostat (Leica Microsystems, Wetzler, Germany). The sections were stained with DAPI to visualize the nuclei and immunolabelled with antibodies against PECAM1 (1:200), Tuj1 (1:100), Iba1 (1:300) and GFAP (1:200) to visualize BCECs, neurons, microglia and astrocytes, respectively. Next, the sections were incubated with an Alexa Fluor 488-conjugated secondary antibody. All images were acquired using the A1R confocal laser-scanning microscope (Nikon Corp.).

Data availability. The data that support the findings reported herein are available on reasonable request from the corresponding authors.

Received: 9 March 2017 Accepted: 7 August 2017

Published online: 17 October 2017

\section{References}

1. Cabral, H. et al. Accumulation of sub- $100 \mathrm{~nm}$ polymeric micelles in poorly permeable tumours depends on size. Nat. Nanotechnol 6, 815-823 (2011).

2. Yoo, J. W. \& Mitragotri, S. Polymer particles that switch shape in response to a stimulus. Proc. Natl Acad. Sci. USA 107, 11205-11210 (2010).

3. Geng, Y. et al. Shape effects of filaments versus spherical particles in flow and drug delivery. Nat. Nanotechnol 2, 249-255 (2007).

4. Mitragotri, S., Burke, P. \& Langer, R. Overcoming the challenges in administering biopharmaceuticals: formulation and delivery strategies. Nat. Rev. Drug Discov. 13, 655-672 (2014).

5. Petros, R. A. \& DeSimone, J. M. Strategies in the design of nanoparticles for therapeutic applications. Nat. Rev. Drug Discov. 9, 615-627 (2010).

6. Napoli, A. et al. Oxidation-responsive polymeric vesicles. Nat. Mater. 3, 183-189 (2004)

7. Ferrari, M. Cancer nanotechnology: opportunities and challenges. Nat. Rev. Cancer 5, 161-171 (2005).

8. Matsumura, Y. \& Maeda, H. A new concept for macromolecular therapeutics in cancer chemotherapy: mechanism of tumoritropic accumulation of proteins and the antitumor agent smancs. Cancer Res. 46, 6387-6392 (1986).

9. Nishiyama, N. et al. Novel cisplatin-incorporated polymeric micelles can eradicate solid tumors in mice. Cancer Res. 63, 8977-8983 (2003).

10. Duncan, R. The dawning era of polymer therapeutics. Nat. Rev. Drug Discov. 2, 347-360 (2003)

11. Kim, B. Y., Rutka, J. T. \& Chan, W. C. Nanomedicine. N. Engl. J. Med. 363, 2434-2443 (2010)

12. Plummer, R. et al. A phase I clinical study of cisplatin-incorporated polymeric micelles (NC-6004) in patients with solid tumours. Br. J. Cancer 104, 593-598 (2011).

13. Hamaguchi, T. et al. A phase I and pharmacokinetic study of NK105, a paclitaxel-incorporating micellar nanoparticle formulation. Br. J. Cancer 97, 170-176 (2007)

14. Cabral, H. \& Kataoka, K. Progress of drug-loaded polymeric micelles into clinical studies. J. Control. Release 190, 465-476 (2014).

15. Abbott, N. J., Patabendige, A., Dolman, D. E., Yusof, S. R. \& Begley, D. J. Structure and function of the blood-brain barrier. Neurobiol. Dis. 37, 13-25 (2010).

16. Banks, W. A. From blood-brain barrier to blood-brain interface: new opportunities for CNS drug delivery. Nat. Rev. Drug. Discov. 15, 275-292 (2016).
17. Wiley, D. T., Webster, P., Gale, A. \& Davis, M. E. Transcytosis and brain uptake of transferrin-containing nanoparticles by tuning avidity to transferrin receptor. Proc. Natl Acad. Sci. USA 110, 8662-8667 (2013).

18. Zensi, A. et al. Albumin nanoparticles targeted with Apo E enter the CNS by transcytosis and are delivered to neurones. J. Control. Release 137, 78-86 (2009).

19. Uchida, Y. et al. Quantitative targeted absolute proteomics of human blood-brain barrier transporters and receptors. J. Neurochem. 117, 333-345 (2011).

20. Xie, F. et al. Investigation of glucose-modified liposomes using polyethylene glycols with different chain lengths as the linkers for brain targeting. Int. J. Nanomed. 7, 163-175 (2012).

21. Qin, Y. et al. In vitro and in vivo investigation of glucose-mediated braintargeting liposomes. J. Drug Target. 18, 536-549 (2010).

22. Lodish H., et al. in Molecular Cell Biology, 4th edn (W. H. Freeman, 2000).

23. Underhill, C. B. \& Toole, B. P. Physical characteristics of hyaluronate binding to the surface of simian virus 40-transformed 3T3 cells. J. Biol. Chem. 255, 4544-4549 (1980).

24. Mammen, M., Choi, S. K. \& Whitesides, G. M. Polyvalent interactions in biological systems: Implications for design and use of multivalent ligands and inhibitors. Angew. Chem. Int. Ed. 37, 2754-2794 (1998).

25. Alexis, S. B., Pavel, I., Felipe, Z., Juan, C. V. \& Jorge, F. Predicting the threedimensional structure of the human facilitative glucose transporter glut1 by a novel evolutionary homology strategy: Insights on the molecular mechanism of substrate migration, and binding sites for glucose and inhibitory molecules. Biophys. J. 87, 2990-2999 (2004).

26. Barnett, J. E. G., Holman, G. D. \& Munday, K. A. Structural requirements for binding to the sugar-transport system of the human erythrocyte. Biochem. J. 131, 211-221 (1973).

27. Simpson, I. A. et al. Blood-brain barrier glucose transporter: Effects of hypoand hyperglycemia revisited. J. Neurochem. 72, 238-247 (1999).

28. Matsumoto, Y. et al. Direct and instantaneous observation of intravenously injected substances using intravital confocal micro-videography. Biomed. Opt. Express 1, 1209-1216 (2010).

29. Muoio, V., Persson, P. B. \& Sendeski, M. M. The neurovascular unit - concept review. Acta Physiol. 210, 790-798 (2014).

30. Gerhart, D. Z., LeVasseur, R. J., Broderius, M. A. \& Drewes, L. R. Glucose transporter localization in brain using light and electron immunocytochemistry. J. Neurosci. Res. 22, 464-472 (1989).

31. Eyster, C. A. et al. Discovery of new cargo proteins that enter cells through clathrin-independent endocytosis. Traffic 10, 590-599 (2009).

32. Riskin, A., Nannegari, V. H. \& Mond, Y. Acute effectors of GLUT1 glucose transporter subcellular targeting in $\mathrm{CIT}_{3}$ mouse mammary epithelial cells. Pediatr. Res. 63, 56-61 (2008).

33. Grant, B. D. \& Donaldson, J. G. Pathways and mechanisms of endocytic recycling. Nat. Rev. Mol. Cell. Biol. 10, 597-608 (2009).

34. Leto, D. \& Saltiel, A. R. Regulation of glucose transport by insulin: traffic control of GLUT4. Nat. Rev. Mol. Cell. Biol. 13, 383-396 (2012).

35. Foley, K., Boguslavsky, S. \& Klip, A. Endocytosis, recycling, and regulated exocytosis of glucose transporter 4. Biochemistry 50, 3048-3061 (2011)

36. Marchi, N., Granata, T., Ghosh, C. \& Janigro, D. Blood-brain barrier dysfunction and epilepsy: pathophysiologic role and therapeutic approaches. Epilepsia 53, 1877-1886 (2012).

37. Jiang, X. et al. Nanoparticles of 2-deoxy-D-glucose functionalized poly(ethylene glycol)-co-poly(trimethylene carbonate) for dual-targeted drug delivery in glioma treatment. Biomaterials 35, 518-529 (2014).

38. Oldendorf, W. H., Crane, P. D., Lawner, P. M. \& Braun, L. D. Rapid, transient drop in brain glucose after intravenous phloretin or 3-O-methyl-D-glucose. Stroke 14, 388-393 (1983).

\section{Acknowledgements}

This research was supported by a JSPS KAKENHI Grant-in-Aid for Specially Promoted Research (No. 25000006 to K.K.) from the Ministry of Education, Culture, Sports, Science, and Technology (MEXT) of Japan, the Center of Innovation (COI) Program from the Japan Science and Technology Agency (JST) and the Strategic Research Program for Brain Sciences from Japan Agency for Medical Research and Development (AMED). We are grateful to Dr S. Fukuda at the University of Tokyo Hospital and $\mathrm{Mr} \mathrm{H}$. Hoshi at the Research Hub for Advanced Nano Characterization at the University of Tokyo for their valuable support with the TEM measurements. The authors are grateful to Ms S. Ogura, Ms A. Miyoshi and Dr T. Shimada at the University of Tokyo and Dr D. Yui and Ms K. Yoshida-Tanaka at Tokyo Medical and Dental University for technical assistance.

\section{Author contributions}

Y.A., H.K. and Y.F. designed and performed all the experiments. T.I. helped with the polymer synthesis. A.M. and K.N. helped with the biodistribution experiments and immunohistochemical analysis. Y.M. and K.T. assisted with intravital microscopy. Y.A. and H.K. wrote the manuscript. K.M., S.U., K.N., K.O., K.I., N.N., H.M. and T.Ya 
commented on the manuscript. T.Yo. and K.K. edited the manuscript and supervised the whole project.

\section{Additional information}

Supplementary Information accompanies this paper at doi:10.1038/s41467-017-00952-3.

Competing interests: T.Yo. and K.K. are scientific advisors of Braizon Therapeutics, Inc. The remaining authors declare no competing financial interests.

Reprints and permission information is available online at http://npg.nature.com/ reprintsandpermissions/

Publisher's note: Springer Nature remains neutral with regard to jurisdictional claims in published maps and institutional affiliations. (c) (i) Open Access This article is licensed under a Creative Commons Attribution 4.0 International License, which permits use, sharing, adaptation, distribution and reproduction in any medium or format, as long as you give appropriate credit to the original author(s) and the source, provide a link to the Creative Commons license, and indicate if changes were made. The images or other third party material in this article are included in the article's Creative Commons license, unless indicated otherwise in a credit line to the material. If material is not included in the article's Creative Commons license and your intended use is not permitted by statutory regulation or exceeds the permitted use, you will need to obtain permission directly from the copyright holder. To view a copy of this license, visit http://creativecommons.org/ licenses/by/4.0/.

(C) The Author(s) 2017 